\title{
BLOWUP SOLUTIONS TO KELLER-SEGEL SYSTEM AND ITS SIMPLIFIED SYSTEMS
}

\author{
TAKASI SENBA \\ Applied Mathematics, Faculty of Engineering, University of Miyazaki \\ 1-1 Gakuen Kibanadai Nishi, Miyazaki, 889-2192, Japan \\ E-mail:t0f302u@cc.miyazaki-u.ac.jp
}

\begin{abstract}
In this paper, we will consider blowup solutions to the so called Keller-Segel system and its simplified form. The Keller-Segel system was introduced to describe how cellular slime molds aggregate, owing to the motion of the cells toward a higher concentration of a chemical substance produced by themselves. We will describe a common conjecture in connection with blowup solutions to the Keller-Segel system, and some results for solutions to simplified versions of the Keller-Segel system, giving the evidence that the conjecture is true.
\end{abstract}

1. Introduction. In this paper, we will consider blowup of solutions to the so called Keller-Segel system and simplified versions of the Keller-Segel system. Keller and Segel $[\mathrm{KS}]$ introduced a system for describing how cellular slime molds aggregate, owing to the motion of the cells toward a higher concentration of a chemical substance produced by themselves. Nanjundiah [Nan] introduced the system

$$
\left\{\begin{array}{l}
u_{t}=\nabla \cdot(\nabla u-u \nabla v) \text { in } \Omega \times(0, T), \\
v_{t}=\Delta v-v+u \text { in } \Omega \times(0, T), \\
\frac{\partial u}{\partial \nu}=\frac{\partial v}{\partial \nu}=0 \text { on } \partial \Omega \times(0, T), \\
u(\cdot, 0)=u_{0}, v(\cdot, 0)=v_{0} \text { in } \Omega .
\end{array}\right.
$$

as a simplified version of the system introduced by Keller and Segel [KS], where $\Omega$ is a bounded domain in $\mathbf{R}^{N}, N=1,2,3, \cdots$, with smooth boundary $\partial \Omega, \nu(x)$ is the unit normal outer vector on $\partial \Omega$, and $u_{0}$ and $v_{0}$ are smooth and nonnegative in $\bar{\Omega}$.

The system (1) has a unique classical solution $(u, v)$ in $\Omega \times(0, T)$ with some $T>0$, see $[\mathrm{Y}]$. By the maximum principle, we have that the solution $(u, v)$ is nonnegative in $\bar{\Omega} \times(0, T)$. Let $T_{\max }$ be the maximal existence time of the classical solution.

2000 Mathematics Subject Classification: Primary 35K55, 35K57, 92C17; Secondary 35B35, 25B40.

Key words and phrases: chemotaxis, self-similar, blowup.

The paper is in final form and no version of it will be published elsewhere. 
Integrating the first equation of (1) over $\Omega \times(0, t)$ and noticing that $u \geq 0$, we have

$$
\int_{\Omega} u(x, t) d x=\int_{\Omega} u_{0}(x) d x \quad \text { in }\left(0, T_{\max }\right) .
$$

We say that a solution $(u, v)$ blows up at $(q, T)$ if $u(\cdot, t)$ is bounded in $\Omega$ for any $t \in(0, T)$, and if there exists a sequence $\left\{\left(x_{n}, t_{n}\right)\right\} \subset \bar{\Omega} \times(0, T)$ satisfying

$$
\lim _{n \rightarrow \infty}\left(x_{n}, t_{n}\right)=(q, T) \text { and } \lim _{n \rightarrow \infty} u\left(x_{n}, t_{n}\right)=\infty .
$$

Then $q$ and $T$ are a blowup point and the blowup time, respectively. If $T_{\max }<\infty$, we can show that the solution $(u, v)$ blows up at $t=T_{\max }$. That is to say, $T_{\max }$ is the blowup time if $T_{\max }<\infty$.

In the case where $\Omega$ is a bounded domain in $\mathbf{R}$, solutions to (1) do not blow up, see [OY, HP, HW]. That is to say, the following theorem holds.

Theorem 1. If $\Omega=(a, b)$ with $-\infty<a<b<\infty$, each solution ( $u, v)$ to (1) satisfies $T_{\max }=\infty$ and

$$
\sup _{(x, t) \in(a, b) \times(0, \infty)} u(x, t)=\sup _{(x, t) \in(a, b) \times(0, \infty)} v(x, t)<\infty .
$$

Therefore, we shall consider the case where $\Omega \subset \mathbf{R}^{N}$ and $N \geq 2$.

2. Two dimensional case. In this section, we shall consider blowup solutions to (1) and a simplified version of (1) in the domain $\Omega \subset \mathbf{R}^{2}$.

The following theorem was shown by Herrero and Velázquez [HV]

Theorem 2. Let $\Omega=\left\{x \in \mathbf{R}^{2}|| x \mid<L\right\}$ and $L \in(0, \infty)$. Then, there exists a radially symmetric solution to (1) satisfying

$$
u(\cdot, t) \rightarrow 8 \pi \delta_{0}+f \quad \text { in } \mathcal{M}(\bar{\Omega}) \text { as } t \rightarrow T_{\max }
$$

where $T_{\max }<\infty$ and $f$ is a radial and nonnegative function in $L^{1}(\Omega) \bigcap C(\bar{\Omega} \backslash\{0\})$.

We can regard $(u, v)$ as a solution in a half disk $\omega=\left\{x=\left(x_{1}, x_{2}\right) \in \mathbf{R}^{2}|| x \mid<L\right.$, $\left.x_{2}>0\right\}$, since $(u, v)$ satisfies the boundary condition $\partial u / \partial \nu=\partial v / \partial \nu=0$ on $\partial \omega$. Then, Theorem 2 gives that there exists a solution $(u, v)$ to $(1)$ with $\Omega=\omega$ satisfying

$$
u(\cdot, t) \rightarrow 4 \pi \delta_{0}+f \quad \text { in } \mathcal{M}(\bar{\omega}) \text { as } t \rightarrow T_{\max } .
$$

Therefore, we can expect that the singularities in the domain are equal to $8 \pi$ times the delta function and that those on the boundary are equal to $4 \pi$ times the delta function.

In fact, the above holds for blowup solutions to the following system

$$
\left\{\begin{array}{l}
u_{t}=\nabla \cdot(\nabla u-u \nabla v) \quad \text { in } \Omega \times\left(0, T_{\max }\right), \\
0=\Delta v-v+u \quad \text { in } \Omega \times\left(0, T_{\max }\right), \\
\frac{\partial u}{\partial \nu}=\frac{\partial v}{\partial \nu}=0 \quad \text { on } \partial \Omega \times\left(0, T_{\max }\right), \\
u(\cdot, 0)=u_{0} \quad \text { in } \Omega .
\end{array}\right.
$$

Here, $\Omega$ is a bounded domain in $\mathbf{R}^{2}$ with smooth boundary, and $u_{0}$ is smooth and nonnegative.

The system (3) was introduced as a simplified system of (1) by Nagai [Nag]. 
By an argument similar to the one mentioned in Introduction, (2) holds also for solutions to $(3)$, and $u$ is nonnegative in $\Omega \times\left(0, T_{\max }\right)$.

Since the system (3) is similar to the Keller-Segel system, we can expect that the properties of solutions to (3) are similar to those to (1).

The following theorem was shown in [SS] and [Suz].

THEOREM 3. If $T_{\max }<\infty$, the solution $(u, v)$ to (3) satisfies

$$
u(\cdot, t) \rightarrow \sum_{q \in \mathcal{B}} m_{*}(q) \delta_{q}+f \quad \text { in } \mathcal{M}(\bar{\Omega}) \quad \text { as } t \rightarrow T_{\max } .
$$

where

$$
m_{*}(q)= \begin{cases}4 \pi & \text { if } q \in \partial \Omega, \\ 8 \pi & \text { if } q \in \Omega,\end{cases}
$$

$\mathcal{B}$ is the set of blowup points, $\delta_{q}$ is the delta function supported at the point $q$, and $f$ is a nonnegative function in $L^{1}(\Omega) \cap C(\bar{\Omega} \backslash \mathcal{B})$.

Therefore, if $T_{\max }<\infty$, we have $\int_{\Omega} u_{0}(x) d x \geq 4 \pi$.

Considering Theorems 2 and 3, we can expect the following to hold:

A common conjecture in COnneCtion with the Keller-Segel System. If a solution $(u, v)$ to $(1)$ blows up in finite time $T_{\text {max }}$, blowup points are finite and $u$ satisfies

$$
u(\cdot, t) \rightarrow \sum_{q \in \mathcal{B}} m_{*}(q) \delta_{q}+f \quad \text { in } \mathcal{M}(\bar{\Omega}) \quad \text { as } t \rightarrow T_{\text {max }} .
$$

Here, $\mathcal{B}$ is the set of blowup points, $m_{*}$ is the function in $\bar{\Omega}$ in Theorem 3 , and $f$ is a nonnegative function in $L^{1}(\Omega) \cap C(\bar{\Omega} \backslash \mathcal{B})$.

3. Three or more dimensional case. In this section, we shall consider blowup solutions to the Keller-Segel system and its simplified systems in $\Omega \subset \mathbf{R}^{N}$, in the case $N \geq 3$.

First, we shall consider the following example.

ExAmple 1. Let $L \in(0, \infty), D=\left\{x^{\prime}=\left(x_{1}, x_{2}\right) \in \mathbf{R}^{2}|| x^{\prime} \mid<L\right\}, \Omega=\left\{x=\left(x^{\prime}, x_{3}\right)\right.$ $\left.\in \mathbf{R}^{3} \mid x^{\prime} \in D, x_{3} \in(0,1)\right\}$ and let $(\bar{u}, \bar{v})$ be a blowup solution in Theorem 2. Then, $(u(x, t), v(x, t))=\left(\bar{u}\left(x^{\prime}, t\right), \bar{v}\left(x^{\prime}, t\right)\right)$ is a solution to (1) in $\Omega \subset \mathbf{R}^{3}$. By Theorem 2, we obtain that the blowup set is $\{0\} \times(0,1) \subset \Omega$ and that two dimensional delta function appears at each blowup point.

These singularities appear essentially in two dimensional domains.

In order to exclude such singularities from consideration, we shall consider only radially symmetric solutions, in the case $\Omega \subset \mathbf{R}^{N}$ and $N \geq 3$.

Nagai $[\mathrm{Nag}]$ showed the following

Theorem 4. Let $N \geq 3, L \in(0, \infty), \Omega=\left\{x \in \mathbf{R}^{N}|| x \mid<L\right\}$, and let $u_{0}$ be radial and nonnegative. If $u_{0}$ satisfies

$$
\int_{\Omega} u_{0}(x) d x>0 \quad \text { and } \quad \int_{\Omega} u_{0}(x)|x|^{N} d x \ll 1,
$$

then the solution to (3) blows up in finite time. 
Theorem 4 implies that there exist blowup solutions even if $\int_{\Omega} u_{0}(x) d x<4 \pi$. Thus, we can expect that there exist singularities which are not equal to $8 \pi \delta_{q}$ or $4 \pi \delta_{q}$, in the case where $N \geq 3$.

Herrero, Medina and Velázquez [HMV1] introduced the following system as a simplified version of (1):

$$
\left\{\begin{array}{l}
u_{t}=\nabla \cdot(\nabla u-u \nabla v) \quad \text { in } \mathbf{R}^{N} \times\left(0, T_{\max }\right) \\
0=\Delta v-\mu+u \quad \text { in } \mathbf{R}^{N} \times\left(0, T_{\max }\right) \\
\frac{\partial u}{\partial \nu}=\frac{\partial v}{\partial \nu}=0 \quad \text { on } \partial \mathbf{R}^{N} \times\left(0, T_{\max }\right) \\
u(\cdot, 0)=u_{0} \quad \text { in } \mathbf{R}^{N}
\end{array}\right.
$$

where $\mu \geq 0$, and showed the following theorem.

TheOREM 5. For $T>0$ and $m>0$, there exists a radially symmetric solution $(u, v)$ to (4) with $\mu>0$ and $N=3$ blowing up at $x=0$ and $t=T$ and satisfying

$$
\lim _{r \rightarrow 0} \lim _{t \rightarrow T} \int_{|x|<r} u(x, t) d x=m .
$$

Next, we shall consider particular blowing up solutions.

Definition 1 . We say that $(u, v)$ is a (backward) self-similar solution to (4) with $\mu=0$, if there exist functions $\bar{u}$ and $\bar{v}$ in $\mathbf{R}^{N}$ satisfying

$$
(u(x, t), v(x, t))=\left(\frac{1}{T-t} \bar{u}\left(\frac{x}{\sqrt{T-t}}\right), \bar{v}\left(\frac{x}{\sqrt{T-t}}\right)\right)
$$

for a $T>0 .(\bar{u}, \bar{v})$ is called a profile function.

The following theorem was shown by Herrero, Medina and Velázquez [HMV2] and the author [S1] in the case $N=3$ and $N \geq 4$, respectively.

THEOREM 6 . In the case $3 \leq N \leq 9$, there exist radial profile functions $(\bar{u}, \bar{v})$ satisfying $\bar{u} \in L^{\infty}\left(\mathbf{R}^{N}\right), \bar{u}>0$,

$$
\bar{v}(x)=-\int_{0}^{|x|} \frac{1}{\omega_{N} r^{N-1}} \int_{|\tilde{x}|<r} \bar{u}(\tilde{x}) d \tilde{x} d r
$$

and

$$
|x|^{2} \bar{u}(x) \rightarrow C_{s e} \quad \text { as }|x| \rightarrow \infty
$$

with some $C_{s e}>0$.

In the case where $N \geq 10$, there exists a radial profile function $(\bar{u}, \bar{v})$ satisfying $\bar{u} \in$ $L^{\infty}\left(\mathbf{R}^{N}\right), \bar{u}>0$, (5) and (6) with $C_{\text {se }}=4(N-2)$.

For $T>0$ and a profile function $(\bar{u}, \bar{v})$ in Theorem 4 , the corresponding self-similar solution $(u(x, t), v(x, t))=\left((T-t)^{-1} \bar{u}(x / \sqrt{T-t}), \bar{v}(x / \sqrt{T-t})\right)$ blows up at $x=0$ and $t=T$ and satisfies

$$
\lim _{t \rightarrow T} u(x, t)=\lim _{t \rightarrow T} \frac{1}{|x|^{2}} \cdot\left(\frac{|x|^{2}}{T-t} \bar{u}\left(\frac{x}{\sqrt{T-t}}\right)\right)=\frac{C_{s e}}{|x|^{2}} \quad \text { loc. unif. in } \mathbf{R}^{N} \backslash\{0\} .
$$

We observe that self-similar solutions have singularities different from the delta function.

The author showed in [S2] the following theorem: 
TheOREM 7. Let $N \geq 11$. For any sufficiently small $T>0$ and $\varepsilon>0$, there exists a radially symmmetric solution $(u, v)$ to (4) with $\mu=0$ in $\mathbf{R}^{N}$ blowing up at $x=0$ and $t=T$ and satisfying

$$
\left|u(x, T)-\frac{2(N-2)}{|x|^{2}}\right| \leq \frac{\varepsilon}{|x|^{2}} \quad \text { in } \mathbf{R}^{N} \backslash\{0\},
$$

where $u(x, T)=\lim _{t \rightarrow T} u(x, t)$.

By Theorems 6 and 7 , we obtain that, in the case where $N \geq 11$, at least two kinds of singularities appear in blowup solutions to (4) with $\mu=0$.

Considering Theorems 4, 5, 6 and 7, we can expect that for $N \geq 3$ many kinds of singularities appear in blowup solutions to the Keller-Segel system.

\section{References}

[HP] T. Hillen and A. Potapov, The one-dimensional chemotaxis model: global existence and asymptotic profile, Math. Methods Appl. Sci. 27 (2004), 1783-1801.

[HMV1] M. A. Herrero, E. Medina and J. J. V. Velázquez, Finite-time aggregation into a single point in a reaction diffusion system, Nonlinearity 10 (1997), 1739-1754.

[HMV2] M. A. Herrero, E. Medina and J. J. V. Velázquez, Self-similar blow-up for a reaction diffusion system, J. Computational and Applied Mathematics 97 (1998), 99-119.

[HV] M. A. Herrero and J. J. V. Velázquez, A blow-up mechanism for a chemotaxis model, Ann. Scuola Norm. Sup. 24 (1997), 633-683.

[HW] D. Horstmann and M. Winkler, Bounded vs. blow-up in a chemotaxis system, J. Differential Equations 215 (2005), 52-107.

[KS] E. F. Keller and L. A. Segel, Initiation of slime mold aggregation viewed as an instability, J. Theor. Biol. 26 (1970), 399-415.

[OY] K. Osaki and A. Yagi, Global existence for a chemotaxis-growth system in $\mathbf{R}^{2}$, Adv. Math. Sci. Appl. 12 (2002), 587-606.

[Nag] T. Nagai, Blow-up of radially symmetric solutions to a chemotaxis system, Adv. Math. Sci. Appl. 5 (1995), 581-601.

[Nan] V. Nanjundiah, Chemotaxis, signal relaying and aggregation morphology, J. Theor. Biol. 42 (1973), 63-105.

[S1] T. Senba, Blowup behavior of radial solutions to Jäger-Luckhaus system in high dimensional domains, Funkcialaj Ekvacioj 48 (2005), 247-271.

[S2] T. Senba, A fast blowup solution to an elliptic-parabolic system related to chemotaxis, preprint.

[SS] T. Senba and T. Suzuki, Chemotactic collapse in a parabolic-elliptic system of mathematical biology, Advances in Differential Equations 6 (2001), 21-50.

[Suz] T. Suzuki, Free Energy and Self-Interacting Particles, PNLPDE 62, Birkhäuser, Boston, 2005.

[Y] A. Yagi, Norm behavior of solutions to a parabolic system of chemotaxis, Math. Japonica 45 (1997), 241-265. 
